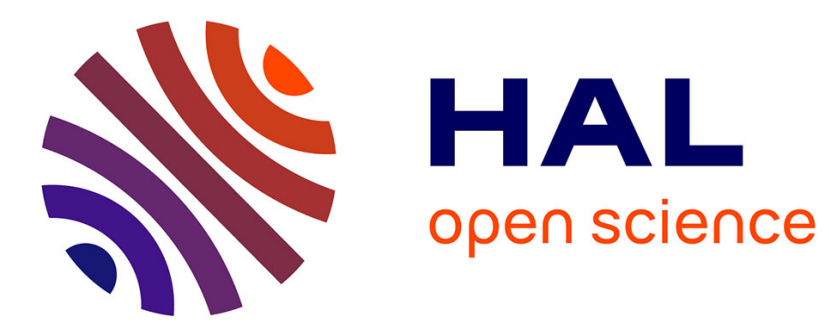

\title{
Unified mathematical framework for a compact and fully parallel n-D skeletonization procedure
}

Antoine Manzanera, Thierry Bernard, Françoise Prêteux, Bernard Longuet

\section{To cite this version:}

Antoine Manzanera, Thierry Bernard, Françoise Prêteux, Bernard Longuet. Unified mathematical framework for a compact and fully parallel n-D skeletonization procedure. Vision Geometry, Jul 1999, Denver, United States. 10.1117/12.364113 . hal-01245457

\section{HAL Id: hal-01245457 \\ https://hal.science/hal-01245457}

Submitted on 17 Dec 2015

HAL is a multi-disciplinary open access archive for the deposit and dissemination of scientific research documents, whether they are published or not. The documents may come from teaching and research institutions in France or abroad, or from public or private research centers.
L'archive ouverte pluridisciplinaire HAL, est destinée au dépôt et à la diffusion de documents scientifiques de niveau recherche, publiés ou non, émanant des établissements d'enseignement et de recherche français ou étrangers, des laboratoires publics ou privés. 


\title{
A unified mathematical framework for a compact and fully parallel n-D skeletonization procedure
}

\author{
Antoine Manzanera ${ }^{a c d}$, Thierry M. Bernard ${ }^{b}$ Françoise Prêteux $^{c}$, and Bernard Longuet ${ }^{a}$ \\ ${ }^{a}$ Aérospatiale E/SCS/V , 2 Rue Béranger, 92320 Chatillon Cedex, France \\ ${ }^{b}$ ENSTA/LEI, 32 Bd Victor, 75015 Paris, France \\ ${ }^{c}$ ARTEMIS Project Unit, INT, 9 Rue Charles Fourier, 91011 Evry Cedex, France \\ ${ }^{d}$ DCE/ETC4/CTA/GIP, 16bis Avenue Prieur de la Côte d'Or, 94114 Arcueil Cedex, France
}

\begin{abstract}
We present in this paper a generic algorithm to compute the skeleton of an $n$-dimensional binary object. Considering the cartesian hypercubic grid, we provide a mathematical framework in which are given the explicit Boolean conditions under which the iterative thinning procedure removes a point. This algorithm preserves the topology in a sense which matches the properties usually used in 2D and 3D. Furthermore, it is based on an original kind of median hypersurface that gives to the skeleton good behavior with respect to both shape preservation and noise sensitivity. The algorithm is fully parallel, as no spatial subiterations are needed. The latter property, together with the symmetry of the boolean $n$-dimensional patterns leads to a perfectly isotropic skeleton. The logical expression of the algorithm is extremely concise, and in 2D, a large comparative study shows that the overall number of elementary Boolean operations needed to get the skeleton is smaller than for the other iterative algorithms reported in the literature.
\end{abstract}

Keywords: Skeleton, Fully Parallel Algorithm, n-dimensional, Discrete Topology, Boolean Complexity

\section{INTRODUCTION}

Representing a shape with a small amount of information is a major challenge in computer vision. Skeletonization is one of the approaches to this purpose. It arises from the idea that a shape is faithfully represented if its topology (connected components, holes...), as well as its geometry (elongated parts, ramifications...) and location are preserved. The interest in skeleta for digital images is motivated by the usefulness of this representation as a preprocessing step in pattern recognition algorithms. A large number of papers has been published in the subject, for 2D images for several decades, and for $3 \mathrm{D}$ images a little more recently. Skeletonization in $\mathrm{nD}$ seems to be a more exotic issue, but it is of some interest in robotics, where it provides the safest trajectories in a multi-parameter space. The skeleton is usually obtained through an iterative procedure called thinning: the border points are removed as long as they are not judged significant for the features stated above, until no more point can be deleted. The remaining shape is then called skeleton. We believe that the available knowledge about digital skeleta suffers two important problems. Firstly, the characterizations that are given in $2 \mathrm{D}$ are difficult to adapt to $3 \mathrm{D}$, which shows the need for unifying concepts. Secondly, these characterizations are complicated, and in particular in $3 \mathrm{D}$, never easy to implement. We propose in the present paper a new thinning algorithm, called MB, which is defined in the hypercubic grid, independently of the dimension. Indeed, the conditions under which a point is removed are defined by means of one removing condition (Function Alpha) and one remaining condition (Function Beta), that are characterized in an adimensional way. Getting the Boolean characterization of the thinning process in any dimension is then straightforward, and we provide the corresponding pattern matching form for dimensions 1 to 3 . Under this form, our procedure lends itself easily to comparison, and its compactness can then be appreciated. Indeed, in 2D, the overall number of elementary Boolean operations needed to compute the skeleton has proved, through a large study, to be lower than for all other thinning algorithm we know of. In 3D, such a study is still to be done, but the conciseness of the Boolean definition, that makes it straightforward to implement, yet shows up. This paper is organised as follows. Section 2

E-mail addresses: Antoine.Manzanera@etca.fr, Thierry.Bernard@ensta.fr, Francoise.Preteux@int-evry.fr, Bernard.Longuet@missiles.aerospatiale.fr 
provides the little theoretical background that is used in the other sections. We next present our thinning algorithm, illustrating with some results. In Section 4 we present the properties of the MB algorithm, in the following order: (1) Isotropy and full parallelism (2) Topology preservation (3) Median hypersurface preservation (4) Noise sensitivity and reconstructibility (5) Computational speed.

\section{PRELIMINARIES}

\subsection{The hypercubic grid.}

Let $Z$ be the set of integers, $N$ the set of natural integers, $R$ the set of real numbers. Let $\mathcal{P}(A)$ be the collection of all subsets of $A, \mathcal{P}^{*}(A)=\mathcal{P}(A) \backslash \emptyset$. Let $n \in N, Z^{n}$ is the $n$-dimensional discrete space. The cubic grid is defined by the immersion of $Z^{n}$ in the affine space attached to $R^{n}$ by means of the following application:

$$
\begin{aligned}
\Phi: \quad Z^{n} & \longrightarrow \mathcal{P}\left(R^{n}\right) \\
z & \longmapsto
\end{aligned}
$$

Thus a point $z$ of the discrete space is identified to an hypercube of the quantified affine space, i.e. to the cartesian product of the closed segments centered around $z, z_{i}$ being the i-th coordinate of $z$ in the canonic basis.

\subsection{Discrete hybercubic topologies.}

Let $\Psi$ be the function defined on $\mathcal{P}^{*}\left(R^{n}\right)$ by $\Psi(P)=\mathcal{V}$ such that $\mathcal{V}$ is the linear manifold generated by $P$. Then $n$ kinds of adjacency relations (and then topologies as well) can be defined on the hypercubic mesh, as follows:

Definition Let $z$ and $z^{\prime}$ be two points of $Z^{n}$ such that $\Phi(z) \cap \Phi\left(z^{\prime}\right) \neq \emptyset$.

then $z$ and $z^{\prime}$ are $k$-adjacent $(0 \leq k \leq n)$ if and only if:

$$
\operatorname{dim}\left(\Psi\left(\Phi(z) \cap \Phi\left(z^{\prime}\right)\right)=k\right.
$$

Definition Let $z$ and $z^{\prime}$ be two points of $Z^{n}$. $z$ and $z^{\prime}$ are $k$-neighbors if there exists $j, k \leq j \leq n$, such that $z$ and $z^{\prime}$ are $j$-adjacent.

The adjacency of two points corresponds then to a non-empty intersection of two hypercubes, and the level of adjacency, to the dimension of the manifold generated by the intersection. Please note the difference with the notion of neighbor, which matches the definitions usually given in the literature. An example in 2D is provided on Figure 1.

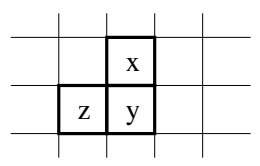

Figure 1. Connectivity relations in dimension 2: $\mathrm{x}$ and $\mathrm{y}$ are 1 -adjacent (and not 0 -adjacent). $\mathrm{x}$ and $\mathrm{z}$ are 0 -adjacent. $\mathrm{x}$ and $\mathrm{y}$ are 1-neighbors, therefore 0-neighbors.

Let a binary image $I$ be a subset of $Z^{n}$.

Definition Interior points of a binary image: $I \subset Z^{n} . z \in I, z$ is a $k$-interior point of $I$ if and only if: $\forall z^{\prime}, z$ and $z^{\prime}$ are $k$-neighbors $\Rightarrow z^{\prime} \in I$.

Counting the $k$-neighbors: Considering the origin $O$ of $Z^{n}$, a point is $k$-adjacent to $O$ if and only if it has $n-k$ coordinates in the set $\{-1,+1\}$, and the others equal to 0 . Thus a point has $A(n, k) k$-adjacent points, with:

$$
A(n, k)=2^{n-k} C_{n}^{n-k}
$$


and then $V(n, k)$ neighbors (itself excluded), with:

$$
V(n, k)=\sum_{i=k}^{n-1} A(n, i)
$$

Table 1 gives the $V(n, k)$ numbers for $n$ smaller than 4 . For $k=0$, we meet of course the overall number of neighbors in dimension $n$ :

$$
W_{n}=V(n, 0)=\sum_{i=0}^{n-1} 2^{n-i} C_{n}^{n-i}=3^{n}-1
$$

We present these numbers to point out the fact that, in the literature, the terms that are usually employed are

Table 1. Numbers of $k$-neighbors in $\mathrm{nD}$, for $n \leq 4$.

\begin{tabular}{c|c|c|c|c}
$\mathrm{k} \backslash \mathrm{n}$ & 1 & 2 & 3 & 4 \\
\hline 3 & - & - & - & 8 \\
\hline 2 & - & - & 6 & 32 \\
\hline 1 & - & 4 & 18 & 64 \\
\hline 0 & 2 & 8 & 26 & 80
\end{tabular}

$V(n, k)$-connectivity, $V(n, k)$-neighbors, etc. Nevertheless, in this paper, with intent to meet both homogeneity and concision, we will always use the single $k$ - prefix.

\subsection{Discrete distances and median hypersurfaces}

Let $\delta_{k}^{n}$ denote the distance induced by the $k$-th topology in dimension $n$. (or $\delta_{k}$ when there is no ambiguity regarding the dimension). Let $X \subset Z^{n}$. Let $X^{c}$ denote $Z^{n} \backslash X$, the background of $X$.

Definition The distance map associated with $X$ and relative to $\delta_{k}$ is the function that associates $\delta_{k}\left(x, X^{c}\right)$ to every $x$ in $Z^{n}$.

Definition Let $r \in N$. Let $x \in Z^{n}$. Let $\mathcal{B}_{k}(x, r)=\left\{y \in Z^{n} ; \delta_{k}(x, y) \leq r\right\}$ be the ball of centre $x$ and radius $r$. Let $X \subset Z^{n} \cdot \mathcal{B}_{k}(x, r)$ is a maximal ball of $X$ if and only if:

$$
\forall y \in X, \forall q \in N, \mathcal{B}_{k}(y, q) \subset X \Rightarrow \mathcal{B}_{k}(x, r) \not \subset \mathcal{B}_{k}(y, q)
$$

Definition Let $S_{k}(X)$ denote the collection of all the centres of maximal balls of the distance $\delta_{k}$. $S_{k}(X)$ is called the median hypersurface associated with distance $\delta_{k}$.

This notion has been the only sheer definition of the skeleton. ${ }^{1}$ But it does not match the modern notion of skeleton, since, in the general case, $X$ and $S_{k}(X)$ do not have the same topology.

Property Let $x \in X . x$ belongs to $S_{k}(X)$ if and only if for every $y k$-neighbor of $x, \delta_{k}\left(y, X^{c}\right) \leq \delta_{k}\left(x, X^{c}\right)$.

In other words, the collection of the centres of maximal balls is equal to the set of local maxima of the corresponding distance map.

The interest of these notions is to formalize the need to represent the geometry and the location of the shape. Indeed, we will guarantee that the skeleton lies right "at the middle" of the shape for some distance if it contains the median hypersurface for this distance.

\section{THINNING PROCEDURE}

Our thinning procedure is defined by means of two binary functions of the discrete space: function Alpha and function Beta. These two functions are respectively defined in Table 2 and in Table 3. The complete algorithm is given in Table 4 .

To express informally the definition given in Table 2, a point $z$ of $I$ such that $\alpha(z)=1$ must be $k$-adjacent to a $(n-1)$-interior point. If this condition holds, these two points have a non-empty intersection, that generates a linear manifold of dimension $k$. Let us now consider the image of this manifold by the symmetry of centre $z$. It is a parallel linear manifold of dimension $k$, equal to the intersection of $n-k$ affine hyperplanes. Then $\alpha(z)=1$ if all the points 
Table 2. Function Alpha.

Let $I \subset Z^{n}$. Function $\alpha: I \longrightarrow\{0,1\}$ is defined as follows:
Let $z \in I$. IF there exists $k, 0 \leq k \leq n-1$, such that:
$(1) \exists z^{\prime}(n-1)$-interior point, $z$ and $z^{\prime}$ are $k$-adjacent
$(2)\left\{t, t(n-1)\right.$-adjacent to $z$ and $\left.\bigcap_{t} \Psi(\Phi(z) \cap \Phi(t))=\mathcal{S}_{z}\left(\Psi\left(\Phi(z) \cap \Phi\left(z^{\prime}\right)\right)\right)\right\} \subset I^{c}$.
where $\mathcal{S}_{z}$ denotes the symmetry of centre $z$.
THEN $\alpha(z)=1$.
$\operatorname{ELSE} \alpha(z)=0$.

Table 3. Function Beta.

\begin{tabular}{|} 
Let $I \subset Z^{n}$. Function $\beta: I \longrightarrow\{0,1\}$ is defined as follows: \\
Let $z \in I$. IF there exists $k, 0 \leq k \leq n-2$, such that: \\
There exist two couples of $k$-adjacent points $(a, b)$ and $(c, d)$ such that \\
$a, b, c, d$ are all $k$-neighbors of $z$, and such that the two following conditions hold: \\
(1) $\Phi(a) \cap \Phi(b)=\Phi(c) \cap \Phi(d)$ \\
(2) $\{a, b\} \subset I$ and $\{c, d\} \subset I^{c}$ \\
THEN $\beta(z)=1$. \\
$\operatorname{ELSE} \beta(z)=0$.
\end{tabular}

$(n-1)$-adjacents to $z$ and whose intersection with $z$ generates one of those hyperplanes belong to the background of $I$.

To express informally the definition given in Table 3, a point $z$ of $I$ such that $\beta(z)=1$ must contain in its $k$ neighborhood two couples of $k$-adjacent points that have the same intersection, and such that one couple belong to the image, and the other to the background.

Figure 2 displays in a table the patterns matched by the functions Alpha and Beta, for dimensions from 1 to 3 : in

Table 4. The MB algorithm.

Repeat for all points $z \in Z^{n}$ within a parallel framework, until convergence:

IF $\alpha(z)=1$ AND $\beta(z)=0$, REMOVE $z$.

dimension $n$, a point is removed if: (1) it matches one of the pattern $\alpha_{i}$ (or one of the $\pi / 2$ rotated version around one of the axis). Here the gray points belong to the image, the white ones to the background, the black point is the origin of the pattern. (2) it does not contain any pattern $\beta_{i}$ within its $i$-neighborhood.

Some results of the MB algorithm are displayed in 2D on Figure 3, and in 3D on Figure 4. They illustrate the properties and behavior of our thinning procedure, that we develop in the following section.

\section{PROPERTIES AND BEHAVIOR}

\subsection{Isotropy and full parallelism}

In the hypercubic grid, isotropy means that all the directions represented by the $n$ axis must be treated the same way, as well as the two directions on every axis. Isotropy is one of the fundamental constraints on which our algorithm is built. A natural outcome of this constraint is the presence of two-pixels thick surfaces in the skeleta, as it can be seen on Figures 3 and 4 . In the definitions of the two Boolean functions, no direction get a special treatment. This property appears clearly on the pattern matching form of the procedure, as all the patterns are completely symmetrical. With this property, isotropy in the sense we have just defined is guaranteed, as long as all the iterations have exactly 


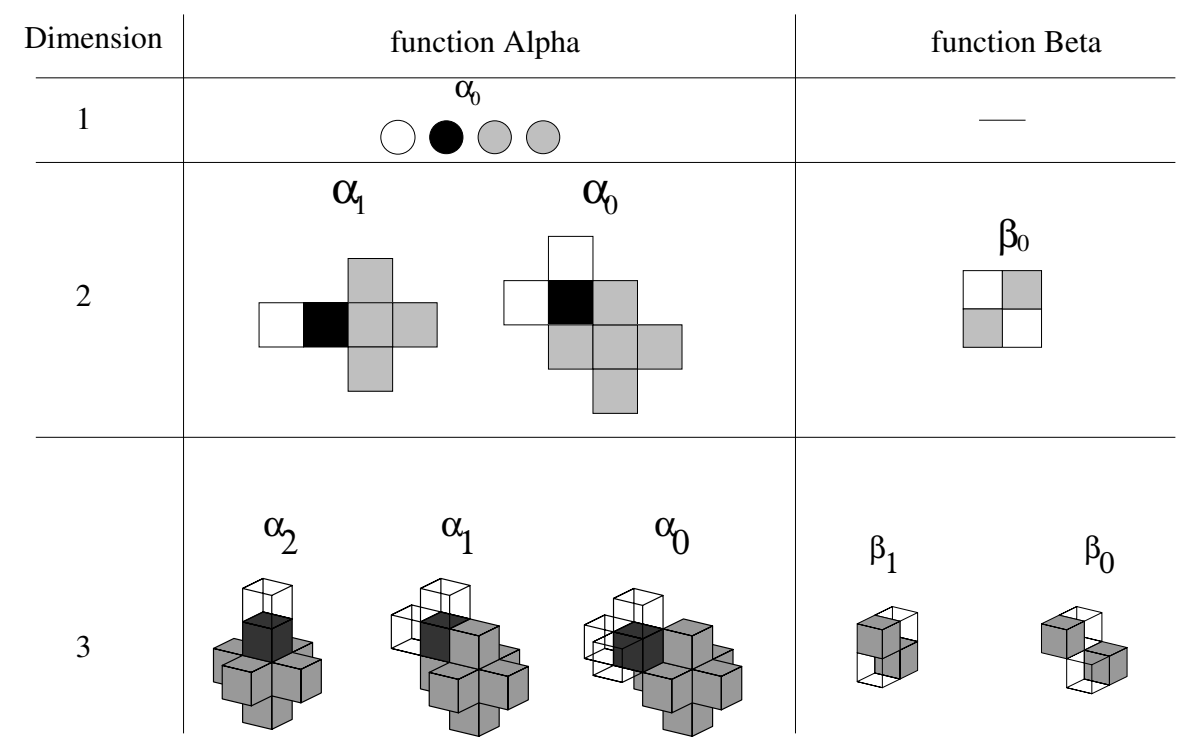

Figure 2. MB patterns in dimension 1 to 3.

the same action. This is an important property of the MB-algorithm: the procedure is fully parallel, which means that all the iterations act exactly the same, removing points in the $2 n$ cardinal directions at the same time. The consequence is that the number of iterations before convergence equals the radius of the biggest ball corresponding to the strongest adjacency relation. As we are going to see in the following subsection, removing points within a parallel framework in all directions while preserving the topology is not trivial, as no Boolean characterization of the points to remove is available, unlike in the case of a sequential framework.

\subsection{Topology preservation}

To remove points from an object without changing its topology means that we aim at preserving the connectivity relations that exist in the object and in its background. We cannot disconnect a connected component, and we cannot create or delete a "hole". Now in a discrete topology, special care must be taken to deal with these notions. For instance, a connected component of the background may run across a piece of surface of the object only if there is a hole in it! To keep this "natural" property meaningful, however, it is necessary to choose two different connectivity levels for the object and its background. In this paper we always consider 0-connectivity for the object (two hypercubes of the object are neighbors as soon as they share a point) and $(n-1)$-connectivity for the background (two hypercubes of the background are neighbors only if they share a face). We use the $(0, n-1)-$ prefix to recall the choice of the topology.

In this section, we do not intend to prove that the MB-algorithm preserves the topology in n-D, as, to our knowledge, no characterization in $\mathrm{n}-\mathrm{D}$ has been recognized by the discrete topology community. Nevertheless, the formalism that we use in the following results is completely generic as for the dimension, and the results are true for dimensions 2 and 3.

We must first give some meaning to "preserving the topology". In the 2D and 3D continuous spaces, topology can be represented by means of the fundamental group, which is the group of the equivalent classes of homotopic arcs (two arcs are said to be homotopic if there exists a continuous function mapping one to the other). Then two subsets of $R^{2}$ have the same topology if and only if their fundamental groups are isomorphic; in $R^{3}$ this property is no longer sufficient, as fundamental groups of the backgrounds must be isomorphic too. In the next dimensions, homotopy groups of higher orders are necessary to characterize the topology.

In discrete spaces, the central notion regarding topology preservation is simplicity. A subset $A$ of a discrete image $X$ is said to be simple if $X$ and $X \backslash A$ have the same topology. For a point $x \in X$, " $x$ is simple" means that $\{x\}$ is simple. In $2 \mathrm{D}$ and $3 \mathrm{D}$ there are explicit characterizations to decide whether a point is simple or not. A very 


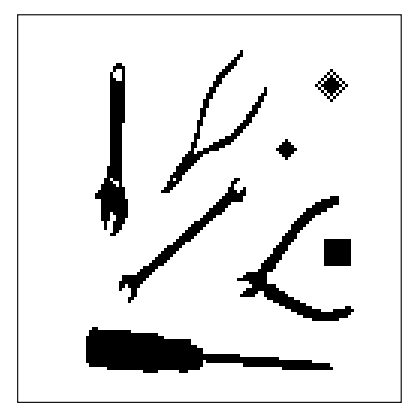

Original

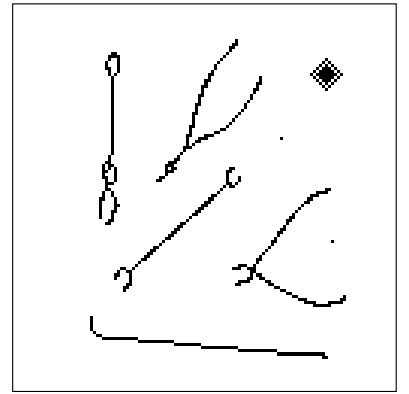

Guo \& Hall: AFP1

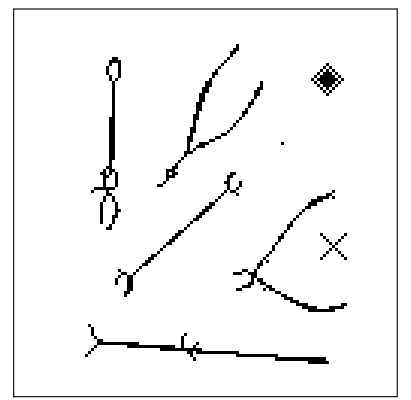

Manzanera \& Bernard

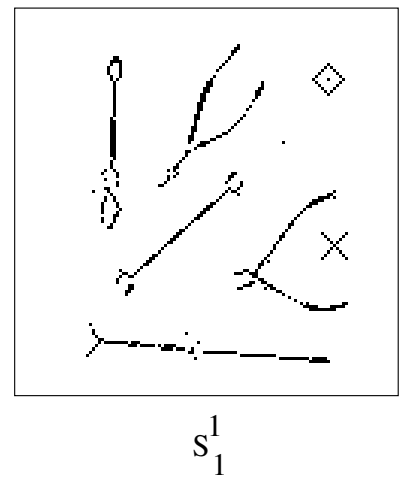

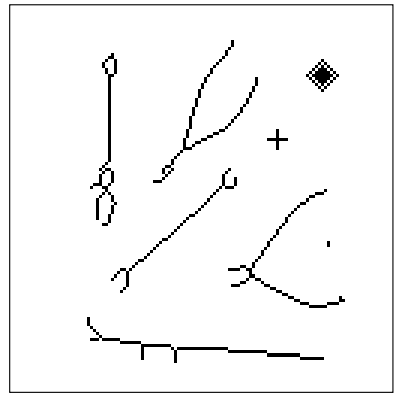

Stewart

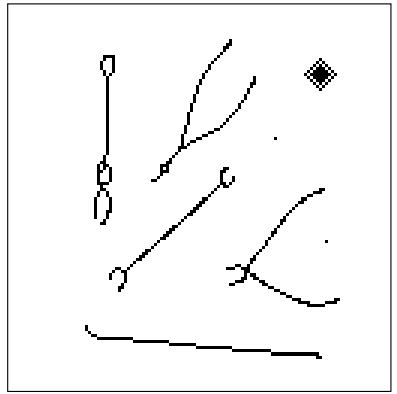

Guo \& Hall: AFP2

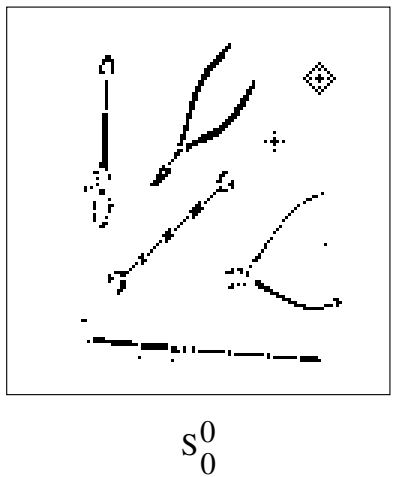

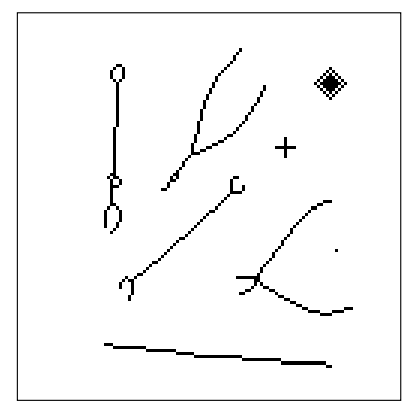

Jang \& Chin

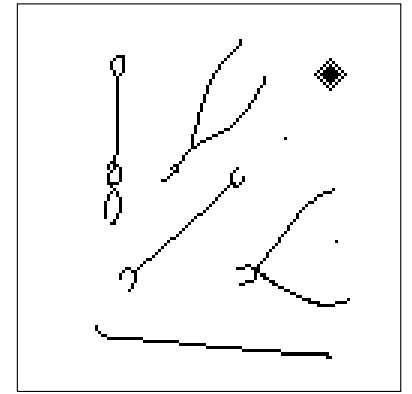

Guo \& Hall: AFP3

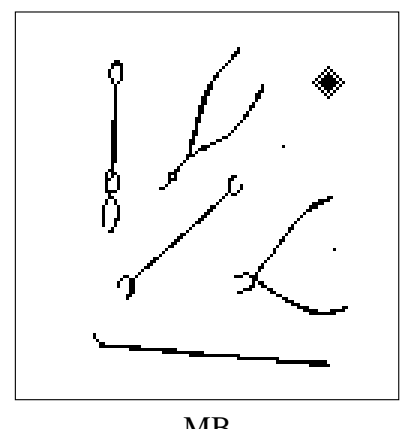

MB

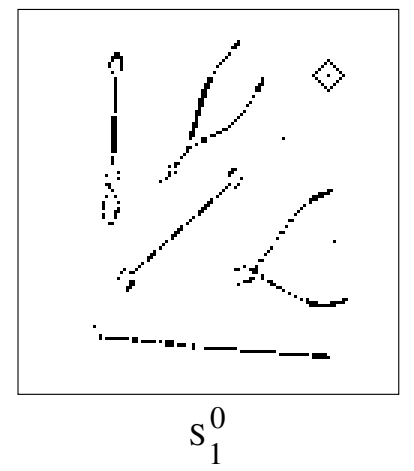

Figure 3. The results of the MB-2D algorithm. For comparison purposes are also displayed the results of the algorithms of Jang and Chin, ${ }^{2}$ Stewart, ${ }^{3}$ the three algorithms of Guo and Hall, ${ }^{4}$ and a previous algorithm of the authors. ${ }^{5}$ The last line shows the different local maxima sets, to be defined in Section 4. 


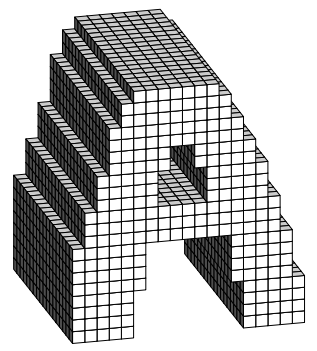

Original

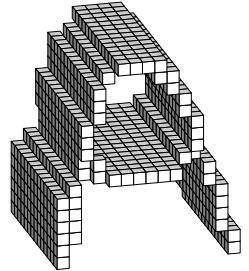

MB-3D

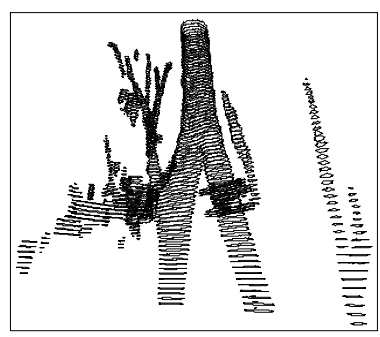

Original

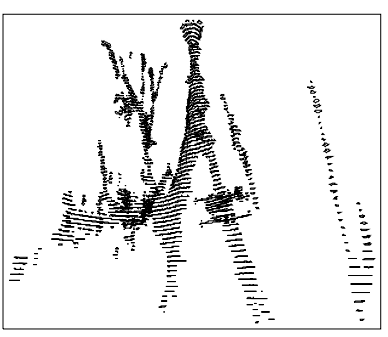

MB-3D

Figure 4. The results of the MB-3D algorithm: on a simple home-made 3D object (1) and on a three-dimensional segmented image of lung (2).

important result is that the decision can be made locally, examining only a finite neighborhood. We now give the characterization, for the $(0, n-1)$-connectivity model:

Theorem Let $X \subset Z^{n}$ be a binary image. Let $x \in X$. Let $X_{0}^{x}$ denote the set of all the 0-neighbors of $x$ in $X$, except $x$ itself, and $\overline{X_{n-2}^{x}}$ the set of all the (n-2)-neighbors of $x$ in $X^{c} . x$ is simple in $X$ for the $(0, \mathrm{n}-1)$-connectivity model if and only if the two following conditions hold:

- $x$ is 0-neighbor with one single 0-connected component of $X_{0}^{x}$.

- $x$ is (n-1)-neighbor with one single (n-1)-connected component of $\overline{X_{n-2}^{x}}$.

In $2 \mathrm{D}$, this result correspond to the 0 -connectivity number that has been defined by Yokoi, ${ }^{6}$ but that had been used earlier by Hilditch. ${ }^{7}$ In $3 \mathrm{D}$, it is a result due to Bertrand and Malandain. ${ }^{8}$ Thus, with the above theorem, we propose a unified expression of those two fundamental results.

Unfortunately, a union of simple points is not a simple set, in general, and this is the major difficulty for designing parallel thinning algorithms. The first problem is to characterize simple sets. Ronse ${ }^{9}$ did it first for $2 \mathrm{D}$ images, it was then generalised by Kong ${ }^{10}$ for higher-dimensional images. In these papers, it is shown that a set is simple for the image $X$ if and only if it can be ordered in a sequence of points $\left\{x_{1}, \ldots, x_{n}\right\}$ such that for every $i$ in $\{1, \ldots, n\}, x_{i}$ is

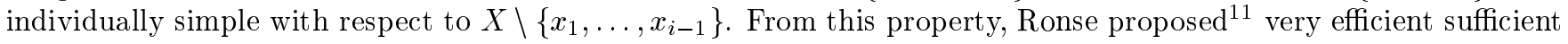
conditions to prove the soundness of parallel thinning algorithm in $2 \mathrm{D}$. This result has been extended to the $3 \mathrm{D}$ case by Ma. ${ }^{12}$ For this topic also, we may give a unified expression of these results as follows: Let a unit lattice element of dimension $k, 0 \leq k \leq n$, be a set of $2^{k}$ points of $Z^{n}$ such that every pair of points is a pair of $(n-k)$-neighbors. Theorem (Ronse 88, Ma 94)

Let $X \subset Z^{n}$ be a binary image. An algorithm that removes points in parallel from a binary nD shape $X$ preserves $(0, \mathrm{n}-1)$-connectivity if the two following conditions are satisfied:

- Every subset of $X$ that is contained in a unit lattice element of dimension $(n-1)$ and that is removed by the algorithm is simple.

- No connected component of $X$ contained in a unit lattice element of dimension $n$ can be completely removed.

This theorem allows to prove the soundness of a parallel thinning algorithm by checking only a limited number of configurations. In this context, the following proposition has been proved for $n=2^{13}$ and for $n=3^{14}$ :

Proposition

MB preserves the $(0, n-1)$-topology. 


\subsection{Median hypersurface conservation}

In this section, we are concerned with the non-topological side of skeletonization, which is geometry conservation. Ideally, we ensure that the skeleton lies at the middle of the shape, if the local maxima of the euclidean distance belong to the skeleton. This distance is, however, complex to compute, and as our former purpose was conciseness, our choice was to deal only with the canonical distances of the hypercubic grid.

Let $p \leq m \leq n$. Let us define the $(m, p)$-median surface as follows:

\section{Definition}

$$
S_{m}^{p}(X)=\left\{x \in X ; \forall y p-n e i g h b o r \text { of } x, \delta_{m}\left(y, X^{c}\right) \leq \delta_{m}\left(x, X^{c}\right)\right\}
$$

Note that the $(m, m)$-median surface of $X$ corresponds to the classical $S_{m}(X)$ defined in Section 2.3. The behavior of MB with respect to median surface preservation is characterized by the following proposition:

Proposition Let $n \in N$. Let $X$ be a well-formed image.

The MB-nD skeleton of $X$ contains the set $S_{n-1}^{0}(X)$.

This important property is illustrated in $2 \mathrm{D}$ on Figure 5 and in 3D on Figure 6. On Figure 5, the upper pictures show the distance functions for the two different connectivity models, where the value is represented by the grey level. On the centre, the left and the right images show the corresponding local maxima set, and at the middle, the original median axis on which the MB skeleton (bottom, centre) is built. For comparison purposes are also shown other skeleta on the left and on the right, based on the local maxima for the 1- and the 0- distance respectively. On Figure 6 , it can be seen as well that different 3D skeleta can be obtained, depending on the median surface. On Figure (b), the restriction of the Alpha function of the MB thinning algorithm to the $\alpha_{2}$ pattern leads to a skeleton based on the local maxima of the 2-distance. On Figure (c), restricting to patterns $\alpha_{2}$ and $\alpha_{1}$ leads to a skeleton based on the $(2,1)$-median surface. Finally, the whole function Alpha is applied on Figure (d), and the resulting skeleton is based on the $(2,0)$-median surface.

We must now tell about the "well-formed images", for which the skeleton contains the $(n-1,0)$-median surface. A well-formed image is an image in which the points are deleted by the thinning operator in the order of the $(n-1)$ distance function. In this case, if we consider the patterns $\alpha_{i}, 0 \leq i \leq n-1$ of Figure 2 , we can see that they can only remove a point that has in its 0 -neighborhood a $(n-1)$-interior point, whose $(n-1)$-distance to the background is strictly superior and thus the points of $S_{n-1}^{0}$ necessarily belong to the skeleton. It is worth observing that, for usual images, points are examined according to the order induced by the distance function. Nevertheless, there are exceptions, like those corresponding to ill-constructed images. We give an example of such an image in $2 \mathrm{D}$ on Figure $7(\mathrm{a})$. These images correspond to a configuration that would "protect" a piece of surface, forbiding a thick volume to be thinned, as in the shape present in the upper right corner of the image on Figure 3.

Although rigorous proof is still to be provided, it seems that a sufficient condition for a n-D image to be well-formed is not to contain one-pixel holes matching one of the patterns $\beta_{i}$. An image $X \subset Z^{n}$ has a one-pixel hole if there exist $x \in X^{c}$ and $\mathcal{V}$ a linear manifold of dimension $k, 2 \leq k \leq n$ such that $x \in \mathcal{V}$ and $\forall y, y$ (n-1)-adjacent to $x, y \in \mathcal{V}$, $y \in X$.

Figure 7 shows the corresponding patterns: a 2D image is well-formed if it does not contain any rotated version of pattern (b), a 3D image if it does not contain any rotated version of patterns (c) or (d), where at least one of the two square points does not belong to $X$.

\subsection{Noise sensitivity and reconstructibility}

The MB skeleton is based on a special kind of median surface, the aim of which is to treat more symmetrically the different kinds of distances in the cubic grid. In particular, the thinning operator does not distinguish the balls of the different distances, producing always one single point for any kind of ball. It also produce one single point for a family of sets that are bounded (for the inclusion) by two balls of different distances, and of the same radius. Let us call these sets Fuzzy balls. We show in Figure 8 some fuzzy balls of radius 7 in $2 \mathrm{D}$, and their skeleton reduced to their centre (superimposed as a white dot). In $2 \mathrm{D}$, it can be proved that a fuzzy ball $B$ (formally defined as a set whose (1,0)-median axis is reduced to a point, called its centre $c$ ) is a set that is included between a 1-ball and a 0-ball, such that for all $x$ in $B, B$ contains the smallest rectangle containing $x$ and $c$.

The behavior of the MB skeleton with respect to fuzzy balls is the cause of its good properties regarding noise immunity and $\pi / 4$ rotation invariance, as can be appreciated on Figure 9, where the results are compared to other algorithms, already referenced in Figure 3. In return, it only allows partial reconstruction. This fact is illustrated on Figure 10: the shape is reconstructed from the weighted skeleton (here, the value of the distance function on the skeleton is represented by the gray level). Unlike the left skeleton (a previous algorithm proposed by the authors ${ }^{5}$ ), 


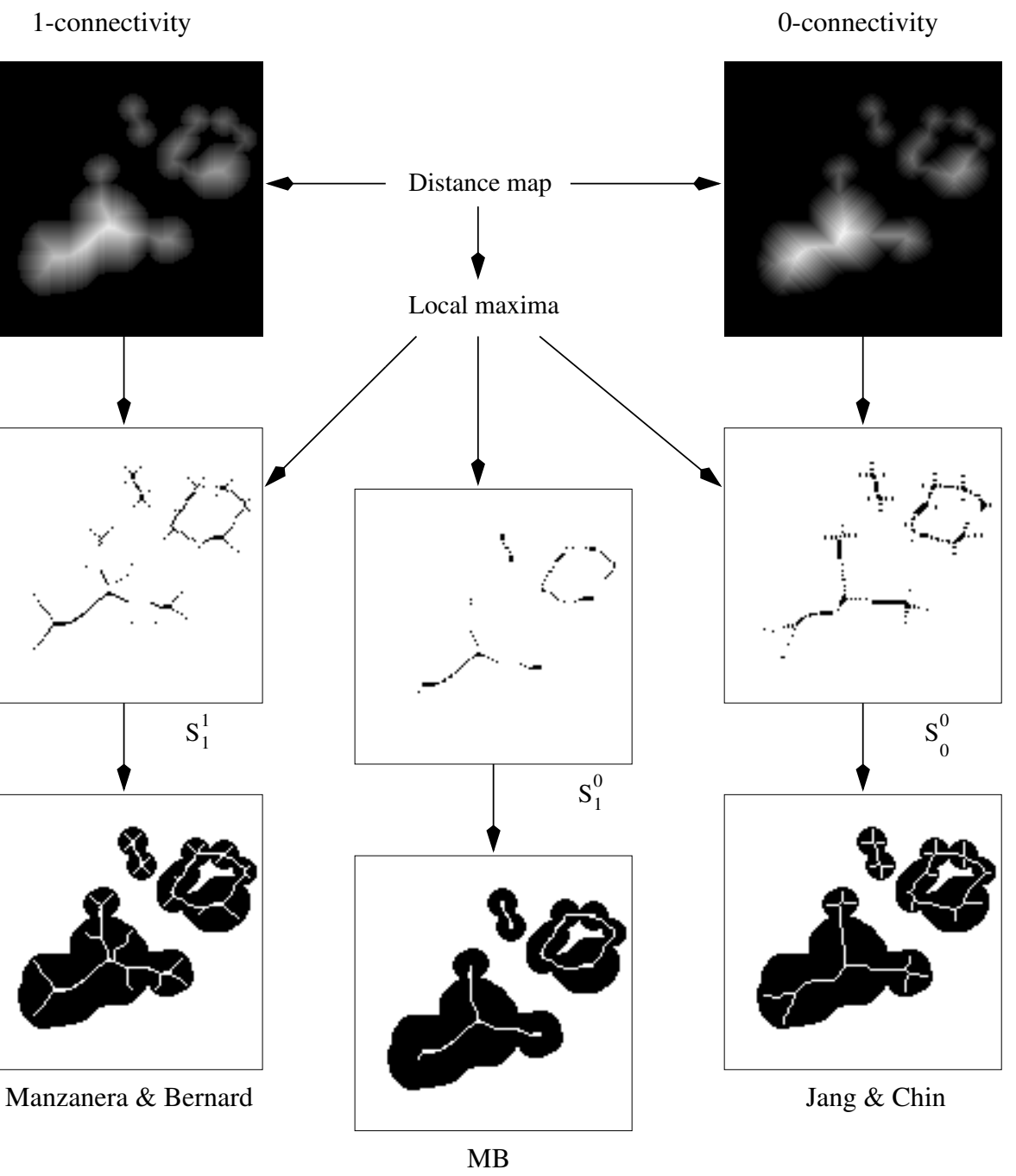

Figure 5. Different median axis leading to different skeleta in 2D.

(a)

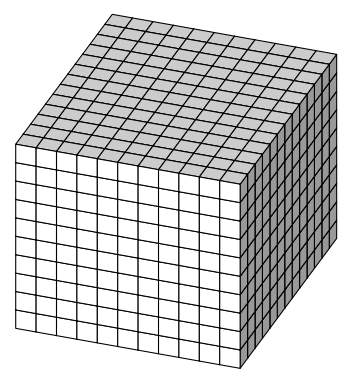

(b)

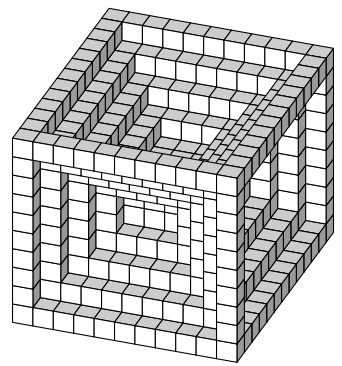

(c)

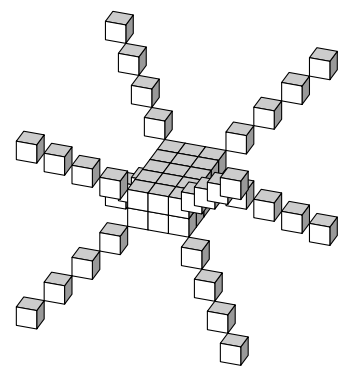

(d)

Figure 6. The different median surfaces in 3D: (a) Original (b) $S_{2}^{2}$ (c) $S_{2}^{1}$ (d) $S_{2}^{0}$. 


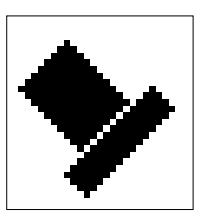

(a)

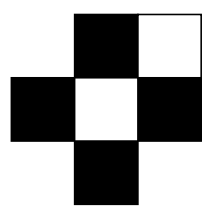

(b)

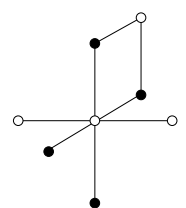

(c)

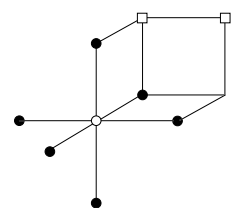

(d)

Figure 7. An ill-constructed image in $2 \mathrm{D}$ (a) and the responsible patterns in $2 \mathrm{D}(\mathrm{b})$ and $3 \mathrm{D}(\mathrm{c}, \mathrm{d})$
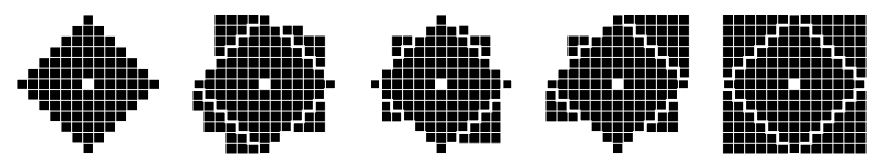

Figure 8. Some fuzzy balls of radius 7 in $2 \mathrm{D}$, and their skeleton reduced to their centre.

that is based on the (1,1)-median axis, and thus allows exact reconstructibility, the MB-algorithm, represented here on the right does not. We show here an approximate reconstruction, based on octagons of the corresponding radius.

\subsection{Computational speed}

Does conceptual conciseness imply computational speed? We have argued in earlier work ${ }^{5}$ that the most fundamental way to compare computational efficiency of data parallel algorithm was by the so-called Shannon measure, i.e. the overall number of elementary operations per pixel needed to perform the algorithm. The nature of the elementary

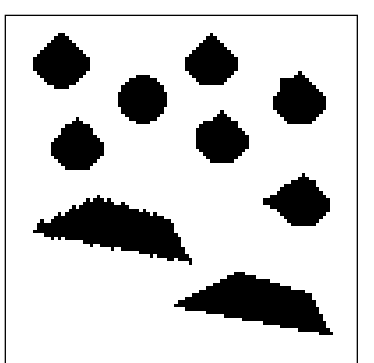

Original

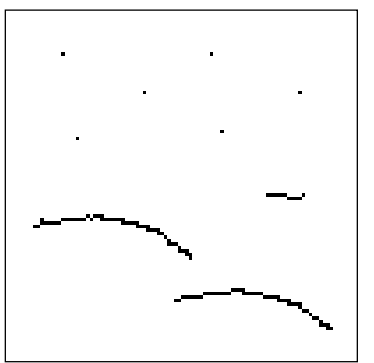

Guo \& Hall: AFP3

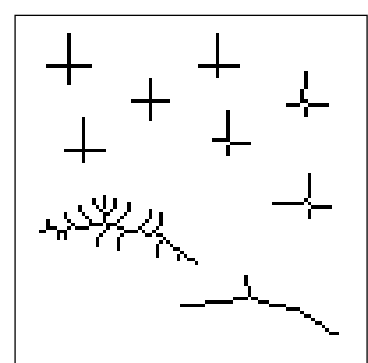

Jang \& Chin

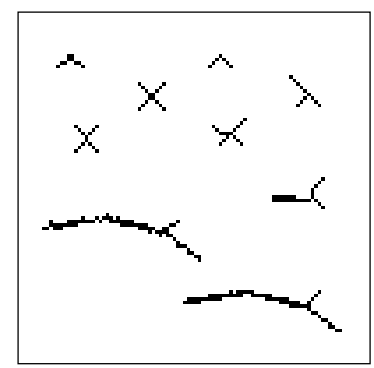

Manzanera \& Bernard

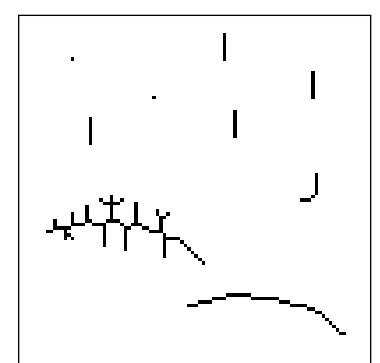

Stewart

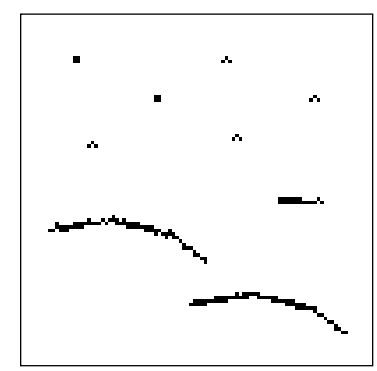

MB

Figure 9. Behavior of the MB algorithm in 2D, with respect to noise. 

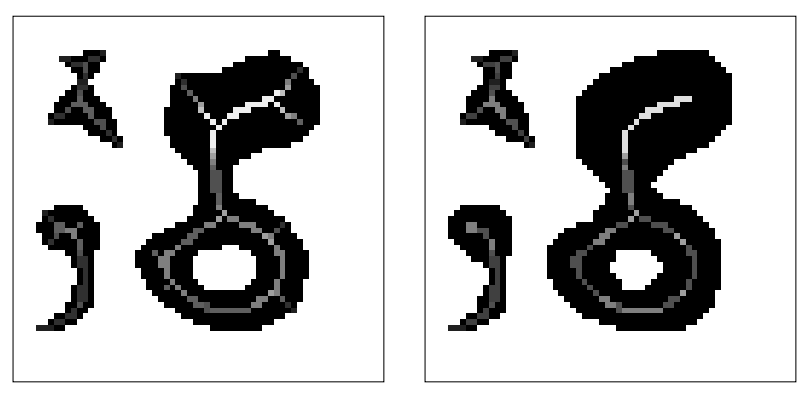

Figure 10. Exact reconstruction for the skeleton based on the 1-distance balls (left), approximate reconstruction for the MB-skeleton based on the fuzzy balls (right).

operations depends on the computer. However, we get a sound general measure by choosing the most elementary operation to be computed in a digital algorithm: the Boolean function of two variables. Indeed, for any digital algorithm, the cost of every elementary Boolean operation is found either at a software level, where it has an effect on the computation time, or at a hardware level, under the form of an equivalent two-entries logic gate, where it has an effect on the expense in hardware resources. The Table 5 displays the Shannon measure of the MB algorithm in $2 \mathrm{D}$ and in $3 \mathrm{D}$. In $2 \mathrm{D}$, the measure is compared to some of the most recent thinning algorithms found in the literature.

Table 5. Comparing the cost of parallel thinning algorithms. $r$ stands for the maximal object thickness (i.e. the radius of the biggest $(n-1)$-ball contained in the image). The number of elementary operations given for the other algorithms is sometimes only an estimation, as the quoted papers did not always provide a logic minimization of their algorithm.

\begin{tabular}{|c|c|c|}
\hline Algorithm & $\begin{array}{c}\text { Size of neighborhood } \\
\text { examined }\end{array}$ & $\begin{array}{c}\text { Number of elementary } \\
\text { operations required }\end{array}$ \\
\hline Jang \& Chin $^{2}$ & 7 & $32 \times r$ \\
\hline Cardoner \& Thomas $^{15}$ & 7 & $40 \times r$ \\
\hline Stewart $^{3}$ & 19 & $64 \times r$ \\
\hline Wu \& Tsai & 11 & $73 \times r$ \\
\hline Guo \& Hall (AFP1) $^{4}$ & 11 & $81 \times r$ \\
\hline Guo \& Hall (AFP2) $^{4}$ & 11 & $91 \times r$ \\
\hline Guo \& Hall (AFP3) $^{4}$ & 11 & $18 \times r$ \\
\hline Manzanera \& Bernard & & $28 \times r$ \\
\hline \hline MB-2D & 13 & $148 \times r$ \\
\hline MB-3D & 21 & \\
\hline
\end{tabular}

\section{CONCLUSION}

The MB thinning algorithm is, to our knowledge, the first thinning algorithm valid both in 2D and in 3D. Further researches in discrete topology for higher dimension meshes will allow to check its overall validity, since the expression in $n-D$ is already available. More generally, we may hope that this work will suggest some research tracks. In particular, we have proposed a unified formalism to express the theorems of homotopy in n-D propositions: the Yokoi/Bertrand and Malandain theorem for the simple point characterization, the Ronse/Ma theorem for the topology preservation sufficient conditions. Do these propositions make sense for dimensions higher than 3 ? One of our goals is to arouse some reactions that will bring us the answer. Nevertheless, we think one of the best achievements of the $\mathrm{MB}$ algorithm is its great simplicity, as the $\mathrm{n}-\mathrm{D}$ thinning procedure is defined through two binary Boolean functions, 
and only $(2 n-1)$ Boolean patterns. We have shown in $2 \mathrm{D}$ that this simplicity had led to computational efficiency, and we can justifiably expect the same advantage in 3D. Finally we have characterized the median hypersurface on which the skeleton is based, and shown that it provided some noise immunity to the skeleton. This median hypersurface implies a new kind of shape descriptors: the fuzzy balls, that we have characterized in 2D. Further works will provide a more general and rigorous view of this last issue.

\section{ACKNOWLEDGMENTS}

The first author is extremely grateful to Bertrand Collin and Damien Mercier, from CTA/GIP, for the visualization tool and software support. The volumetric medical image was acquired in the Service de Radiologie Central de l'Hôpital de la Salpétrière (Professor Ph. Grenier). The lung was segmented by Catalin Fetita from the UP Artemis (INT Evry).

\section{REFERENCES}

1. H. Blum, "A tranformation for extracting new descriptors of shape," in Proc. Symposium Models for the perception of speech and visual form, pp. 62-380, W.Wathen-Dunn ed. M.I.T. Press Cambridge MA, 1967.

2. B.-K. Jang and R. T. Chin, "Analysis of thinning algorithms using mathematical morphology," IEEE Transactions on pattern analysis and machine intelligence 12-6, pp. 514-551, 1990.

3. A. Stewart, "A one-pass thinning algorithm with interference guards," Pattern Recognition Letters 15, pp. 825$832,1994$.

4. Z. Guo and R. W. Hall, "Fast fully parallel thinning algorithms," Computer Vision, Graphics and Image Processing 55-3, pp. 317-328, 1992.

5. A. Manzanera, T. M. Bernard, F. Prêteux, and B. Longuet, "Ultra fast skeleton based on an isotropic fully parallel algorithm," in 8th Discrete Geometry for Computer Imagery, vol. 1568, pp. 313-324, Lecture Notes in Computer Science - Springer Verlag, (Marne-La-Vallée - France), Mar. 1999.

6. S. Yokoi, J. Toriwaki, and T. Fukumura, "Topological properties in digitized binary pictures," Systems, Computers, Controls 4-6, pp. 32-39, 1973.

7. C. Hilditch, "Linear skeletons from square cupboards," Machine Intelligence 4, pp. 403-420, 1969.

8. G. Bertrand and G. Malandain, "A new characterization of three-dimensional simple points," Pattern Recognition Letters 15, pp. 169-175, 1994.

9. C. Ronse, "A topological characterization of thinning," Theoretical Computer Science 43, pp. 31-41, 1986.

10. T. Kong, "On the problem of determining whether a parallel reduction operator for n-dimensional binary images always preserve topology.," in SPIE Conference on Vision Geometry, (Boston - MA), 1993.

11. C. Ronse, "Minimal test patterns for connectivity preservation in parallel thinning algorithms for binary digital images," Discrete Applied Mathematics 21, pp. 67-79, 1988.

12. C. M. Ma, "On topology preservation in 3d thinning," CVGIP: Image Understanding 59-3, pp. 328-339, 1994.

13. T. M. Bernard and A. Manzanera, "Improved low complexity fully parallel thinning algorithm," in (to appear in) International Conference on Image Analysis and Processing, (Venice - Italy), Sept. 1999.

14. A. Manzanera, T. M. Bernard, F. Prêteux, and B. Longuet, "Medial faces from a concise 3d thinning algorithm," in (to appear in) International Conference on Computer Vision, (Kerkyra - Greece), Sept. 1999.

15. R. Cardoner and F. Thomas, "Residuals + directional gaps = skeletons," Pattern Recognition Letters 18, pp. 343-353, 1997.

16. R.-Y. Wu and W.-H. Tsai, "A new one-pass parallel thinning algorithm for binary images," Pattern Recognition Letters 13, pp. 715-723, 1992. 